\title{
VALORACIÓN DE LOS SERVICIOS PÚBLICOS DOMICILIARIOS DE CARTAGENA DE INDIAS (COLOMBIA)*
}

\author{
JUAN CARLOS VERGARA SCHMALBACH" \& FRANCISCO JAVIER MAZA ÁVILA** \\ UNIVERSIDAD DE CARTAGENA - UNIVERSIDAD DEL ZULIA (VENEZUELA)
}

Recibido/ Received/ Recebido: 17/06/2015-Aceptado/ Accepted / Aprovado: 22/07/2016

\begin{abstract}
Resumen
El objetivo de este artículo es valorar la calidad de los servicios públicos domiciliarios en la ciudad de Cartagena de Indias (Colombia), y sus efectos en el nivel de satisfacción de sus habitantes. Para ello se aplicó la técnica de Mínimos Cuadrados Parciales (PLS) a los datos sobre la percepción de la calidad de los servicios públicos domiciliarios (energía, acueducto y alcantarillado, telefonía, internet y gas natural), obtenidos a partir de una encuesta aplicada a una muestra representativa de 250 familias de las 15 comunas de Cartagena de Indias (específicamente adultos responsables del pago de los servicios públicos domiciliarios), utilizando un Muestreo Aleatorio Estratificado. Los resultados arrojaron fuertes correlaciones entre las percepciones de calidad de los cartageneros y su satisfacción frente a los servicios domiciliarios, así como entre las percepciones de calidad y la satisfacción general; esta última relacionada con la valoración de todos los servicios públicos recibidos por los cartageneros, sin importar su género, edad, nivel de formación o estrato socioeconómico. Palabras clave: Servicios públicos; Percepciones; Satisfacción; Hogares; Mínimos Cuadrados Parciales.
\end{abstract}

\section{VALUATION OF DOMESTIC PUBLIC SERVICES IN CARTAGENA DE INDIAS (COLOMBIA)}

\begin{abstract}
The aim of this paper is to assess the quality of public services in the city of Cartagena de Indias (Colombia), and its effects on the level of satisfaction of its inhabitants. For this a Partial Least Squares technique (PLS) was applied to the data on the perception of the quality of public utilities (power, water and sewer, telephone, internet and natural gas), obtained from a survey; and used in a representative selection of 250 families of the 15 communes of Cartagena de Indias (specifically adults responsible for the payment of public services) using a Stratified Random Sampling. The results showed strong correlations between perceptions of quality and satisfaction of the people of
\end{abstract}

Artículo derivado de la investigación titulada "Análisis de la calidad del servicio mediante modelos de Ecuaciones Estructurales" perteneciente a Universidad de Cartagena / Universidad de Zulia.

* Docente de tiempo completo de la Universidad de Cartagena. Ingeniero Industrial (Universidad Tecnológica de Bolívar), Especialista en Finanzas (Universidad de Cartagena), Magister en Administración (Universidad Nacional de Colombia), Candidato a Doctor en Ciencias Sociales mención Gerencia (Universidad de Zulia), Investigador del Grupo Métodos Cuantitativos de Gestión de la Universidad de Cartagena. Correo electrónico: jvergaras@unicartagena.edu.co.

*.* Docente de tiempo completo de la Universidad de Cartagena. Administrador Industrial y Especialista en Gestión Gerencial (Universidad de Cartagena), Magister en Economía y Desarrollo Territorial y Doctor en Ciencias Sociales y Jurídicas (Universidad de Cádiz, España). Director del Grupo de Investigación en Estudios para el Desarrollo Regional -GIDER-. Dirección postal: Universidad de Cartagena, Calle 30 (av. consulado) № 48-15, Cartagena de Indias, Colombia. Teléfono: 6754454. Correo electrónico: fmazaa@unicartagena.edu.co 
Cartagena against domestic services, as well as perceptions of quality and overall satisfaction; the latter related to the valuation of all public services received by the people of Cartagena, regardless of gender, age, education level or socioeconomic status.

Keywords: Public services; Perceptions; Satisfaction; Households; Partial Least Squares.

\title{
AVALIAÇÃO DOS SERVIÇOS PÚBLICOS DOMICILIÁRIOS EM CARTAGENA DE ÍNDIAS (COLÔMBIA)
}

\begin{abstract}
Resumo
O objetivo deste artigo é avaliar a qualidade dos serviços públicos domiciliários na cidade de Cartagena de Índias (Colômbia), e seus efeitos no nível de satisfação de seus habitantes. Para isso se aplicou a técnica de Mínimos Quadrados Parciais (PLS) aos dados sobre a percepção da qualidade dos serviços públicos domiciliários (energia, água e esgotos, telefonia, internet e gás natural), obtidos a partir de uma pesquisa de opinião aplicada a uma mostra representativa de 250 famílias dos 15 bairros de Cartagena de Índias (especificamente adultos responsáveis pelo pagamento dos serviços públicos domiciliários), utilizando uma Amostragem Aleatória Estratificada. Os resultados apresentam fortes correlações entre as percepções de qualidade dos cidadãos e sua satisfação com relação aos serviços domiciliários, bem como entre as percepções de qualidade e a satisfação geral; esta última, relacionada com a avaliação de todos os serviços públicos recebidos pelos moradores da cidade, sem importar seu gênero, idade, nível de formação ou estrato socioeconômico. Palauras chave: Serviços públicos; Percepções; Satisfação; Lares; Mínimos Quadrados Parciais.
\end{abstract}

Vergara, J. \& Maza, F. (2017). Valoración de los servicios públicos domiciliarios de Cartagena de Indias (Colombia). En: Revista de la Facultad de Ciencias Económica: Investigación y Reflexión. rev.fac.cienc.econ, XXV (1). DOI: http://dx.doi.org/10.18359/rfce.2656

JEL: L89, C50.

\section{Introducción}

Dado el alto componente intangible de la prestación de un servicio, su análisis tiende a ser complejo, y aún más cuando se refiere al sector público, ya que no es simplemente una cuestión de satisfacer las necesidades expresadas, sino de descubrir aquellas no expresadas, la identificación de prioridades, y la asignación y justificación de los recursos públicos asignados (Ramseook-munhurrun, Lukea-bhiwajee, \& Naidoo, 2010). Ante esta situación, la calidad de los servicios públicos y la satisfacción de los ciudadanos han sido reconocidos como dos componentes fundamentales para delinear estrategias que permitan reinventar el sector público (Rhee \& Rha, 2009), lo que sugiere que la gestión y propósito de los gobiernos deben derivarse de los ciudadanos, concibiendo el Buen Gobierno como aquel que depende de la calidad de su organización (Giannoccaro, Costantino, Ludovico \& Pietroforte, 2008). De esta manera, una variación en los niveles de satisfacción sobre un servicio público en particular, podría sugerir una variación de la calidad de los servicios ofrecidos (Swindell, Carolina \& Kelly, 2005).

La anterior es la principal razón que lleva a muchos países, a nivel mundial, a aplicar encuestas dirigidas a los ciudadanos para medir las percepciones y el nivel de satisfacción frente al uso de los servicios públicos, aunque dejando a un lado los efectos causales o relaciones que se puedan dar entre estos dos tópicos (Van Ryzin \& Charbonneau, 2010). De 
esta manera, el conocimiento de los efectos que existen entre las percepciones y la satisfacción, se convertiría en un insumo adicional para entender los factores clave que afectan, en mayor medida, el concepto general que tienen los ciudadanos de una localidad sobre los servicios públicos recibidos, para luego delinear acciones centradas específicamente en dichos factores.

Este artículo propone valorar las percepciones que los ciudadanos tienen sobre la calidad de los servicios públicos domiciliarios -energía, acueducto y alcantarillado, telefonía, internet y gas natural- y sus efectos en el nivel de satisfacción, en un caso desarrollado en la ciudad de Cartagena de Indias Colombia-, a través de la aplicación del método de Mínimos Cuadrados Parciales -PLS, por sus siglas en inglés- en un modelo teórico propuesto. Como valor agregado, se propone evaluar el efecto entre el perfil de los encuestados, las percepciones, la satisfacción con un servicio domiciliario en particular y la satisfacción respecto a la valoración general de la calidad de los principales servicios públicos ofrecidos en la ciudad -hospitalarios, domiciliarios, seguridad, infraestructura y educación-. Un análisis individual de los servicios públicos permitirá encontrar los factores clave que inciden, en mayor medida, en la satisfacción de sus usuarios, teniendo en cuenta que los problemas que se encuentran en un servicio a menudo se reproducen en una forma ligeramente diferente en otros tipos de servicios públicos (Ackroyd, Hughes, \& Soothill, 1989). Por ejemplo, los factores clave en los servicios hospitalarios o de seguridad, es probable que sean diferentes a los factores que se encuentran en la educación y los de infraestructura.

Para lograr el objetivo propuesto, se estructura el artículo iniciando con la revisión de la literatura, orientada hacia los referentes teóricos y el análisis de casos similares que giran en torno a la valoración causal de los servicios públicos. Se continúa con la propuesta metodológica y el desarrollo de la investigación en la ciudad de Cartagena de Indias. El artículo finaliza con una discusión centrada en los resultados obtenidos, las conclusiones finales y la bibliografía, indicando (en el caso de que existiese) los códigos DOI de los artículos consultados.

\section{Revisión de la literatura}

La valoración que realizan los usuarios de los servicios públicos recibidos se puede tomar como un referente del desempeño de la gestión administrativa de un gobierno, convirtiéndose en un indicador fundamental en los estudios de satisfacción ciudadana (Christensen, 2002). El desafío no recae directamente en la aplicación de una técnica en particular para comprender los motivantes de la satisfacción; más bien, el éxito de este tipo de estudios está en identificar las variables (latentes o estructurales y sus relaciones) que caracterizan dicha satisfacción respecto a los servicios públicos.

En términos generales, las variables que caracterizan un servicio podrían catalogarse en: percepciones de calidad, satisfacción y comportamientos futuros (Vergara-Schmalbach, Quesada \& Blanco, 2012), cuyas relaciones o efectos causales se pueden resumir en esquema estructural general planteado en la Ilustración 1 (Vergara-Schmalbach, Quesada \& Blanco, 2011).

Entre las percepciones relacionadas con los servicios públicos, se deben considerar aspectos como entrega, acceso o disponibilidad, soporte, información y profesionalismo (Thomson, 2004; Ipsos MORI, 2010). Agus, Barker \& Kandampully (2007), descomponen los servicios públicos en cuatro factores: dimensión, calidad, desempeño y satisfacción; siendo la calidad del servicio un componente que agrupa la mayor parte de las percepciones y que permite, en cierta forma, valorar la gestión de la empresa o entidad prestadora del servicio (Fonseca, 2009). En este sentido, para estos investigadores las percepciones que tienen un mayor impacto en la satisfacción del cliente son: competencia, credibilidad, comunicación, capacidad de respuesta y entendimiento del servicio (Agus et al., 2007). Por su parte, para Ramseook-munhurrun, Lukea-bhiwajee \& Naidoo (2010) las percepciones pueden ser evaluadas a través de la valoración de las dimensiones recomendadas en la escala SERVQUAL: Elementos tangibles, fiabilidad, capacidad de respuesta, seguridad y empatía. 
Ilustración 1. Esquema estructural general para la valoración de la calidad del servicio

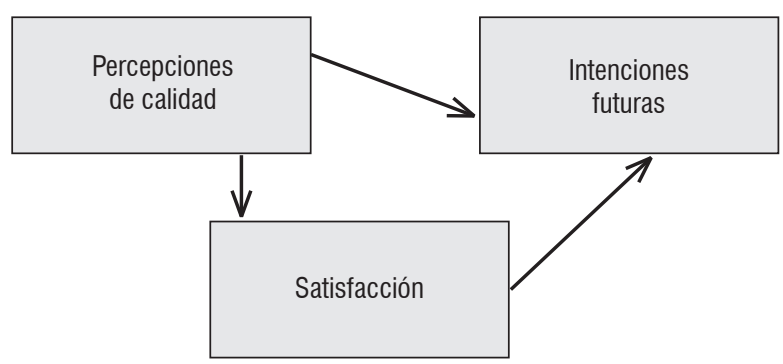

Fuente: Vergara-Schmalbach, Quesada \& Blanco (2011).

En cuanto a las relaciones causales entre percepción y satisfacción, existen diferentes estudios recientes que demuestran un efecto causal directo entre las percepciones sobre los servicios públicos y la satisfacción (James, 2009; Ilhaamie, 2010; Ramseookmunhurrun et al., 2010; Rahaman \& Rahman, 2011; Kondrotaite, 2012; Azizzadeh, Khalili \& Soltani, 2013). Es decir, al mejorar la percepción (o calidad del servicio), se podrá incidir positivamente en diferentes medidas sobre el nivel de satisfacción. Este comportamiento causal también se demuestra entre la satisfacción y las intenciones futuras. Devereux \& Weisbrod (2006), identificaron un fuerte efecto de la satisfacción en dos comportamientos futuros de los usuarios de un servicio público: las quejas y la intención de trasladarse a otro vecindario. Mediante la aplicación de modelos de ecuaciones estructurales -o SEM, por Structural Equation Models-, estos dos investigadores dedujeron que, al mejorar la satisfacción, se podría predecir una disminución de las quejas y el desplazamiento a otra vivienda en otra localidad.

\section{Metodología}

El método de Mínimos Cuadrados Parciales -o PLS, por Partial Least Squares- es una técnica perteneciente a los SEM (Haenlein \& Kaplan, 2004), que combina las características del Análisis de Componentes Principales -ACP- y la Regresión Múltiple (Abdi, 2003) y se emplea en el análisis de las relaciones empíricas de un conjunto de variables, que modelan una situación o supuesto teórico (Rosipal \& Krämer, 2006). Esta técnica es recomendada en situaciones donde el modelo es caracterizado por muchas variables -más de 20 variables independientes- (Garthwaite, 1994) y cuando sólo se dispone de una muestra pequeña -el PLS permite obtener buenos resultados en muestras de tamaño inferior a 50- (Haenlein \& Kaplan, 2004; Mehmood, Martens, Srebø, Warringer \& Snipen, 2011).

\subsection{Modelo estructural}

A partir del esquema propuesto por Vergara-Schmalbach, Quesada \& Blanco (2012), se estructura el siguiente modelo teórico para valorar las percepciones de calidad y satisfacción de los servicios domiciliarios -energía, acueducto y alcantarillado, telefonía, internet y gas natural- en la ciudad de Cartagena, incluyendo elementos del perfil del encuestado y el nivel de satisfacción general, con el fin de enriquecer el análisis causal.

Ilustración 2. Modelo estructural propuesto para la valoración de las percepciones de calidad y satisfacción sobre los servicios públicos domiciliarios en la ciudad de Cartagena de Indias

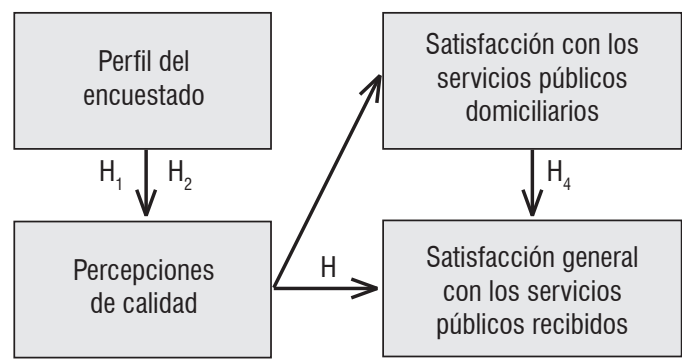

Fuente: Elaboración propia.

En el modelo se identificaron cuatro hipótesis basadas en posibles relaciones causales entre las variables latentes o estructurales presentes:

$H_{1}$ : Existe un efecto causal directo entre el perfil del encuestado y las percepciones de calidad

El perfil corresponde a las características o datos que caracterizan al grupo de ciudadanos encuestados -género, edad, nivel de formación y estrato socioeconómico-, mientras que las percepciones equivalen a la valoración del servicio domiciliario consi- 
derando la calidad, cobertura y acceso. Esta primera hipótesis sugiere la existencia de un efecto causal entre el perfil y la valoración de las percepciones.

$\mathrm{H}_{2}$ : Existe un efecto causal directo entre las percepciones de calidad y la satisfacción con los servicios públicos domiciliarios

$\mathrm{H}_{3}$ : Existe un efecto causal directo entre las percepciones de calidad y la satisfacción general con los servicios públicos recibidos.

La segunda y tercera hipótesis permitirán comprobar la existencia de una correlación positiva entre percepciones de calidad y el nivel de satisfacción -del servicio público domiciliario y de todos los servicios públicos recibidos-, con el fin de identificar los posibles factores clave que mayor incidencia tienen sobre este último.

$\mathrm{H}_{4}$ : Existe un efecto causal directo entre la satisfacción con los servicios públicos domiciliarios y la satisfacción general con los servicios públicos recibidos.

Con esta hipótesis se pretende evaluar el nivel de correlación que existe entre la satisfacción con los servicios domiciliarios y su efecto directo sobre la satisfacción general.

\subsection{Variables y datos}

Para evaluar las variables latentes presentes en el modelo propuesto, se determinaron una serie de variables observables o medibles -identificadas de $\mathrm{P} 1$ a P18-, que se ajustaron al diseño de un cuestionario compuesto por 18 ítems. Por ejemplo, la variable latente denominada como perfil del encuestado, le corresponden cuatro variables observables: género, edad, nivel de formación y estrato socioeconómico. La Tabla 1 muestra las variables empleadas en el modelo, con sus respectivas escalas de valores.

\subsection{Población y muestra}

Se determinó como población el número total de familias con acceso a servicios públicos domiciliarios, equivalente a un tamaño de 177.706 familias, distribuidas en 15 comunas de Cartagena de Indias. Fueron encuestados, bajo un Muestreo Aleatorio Estratificado, 250 adultos responsables del pago de los servicios públicos domiciliarios, estimando un error máximo de $\pm 3,16 \%$ para el estudio.

\subsection{Confiabilidad de la escala}

Para la validar la consistencia del instrumento, se empleó el Alpha de Cronbach, estimador por excelencia para medir la confiabilidad de una escala de valores (Cervantes, 2005). El valor mínimo calculado de este indicador a partir de los datos arrojados por la muestra fue de 0,86, indicando la fiabilidad en las escalas utilizadas en la encuesta (Cortina, 1993).

\section{Resultados}

De la muestra de 250 personas, el 48,8\% fue identificado con sexo Masculino. La mayoría de los encuestados tuvo edades comprendidas entre $20 \mathrm{y}$ 40 años (62\%) y mayores de 60 años (20,8\%). En cuanto al estrato socioeconómico, la mayor parte de los encuestados se ubicó en los estratos 2 -bajoy 3 -medio bajo-, con una proporción de 60,4\% y $20,8 \%$, respectivamente. La Tabla 2 muestra los resultados descriptivos para el total de variables observadas.

El diseño estructural fue analizado con la ayuda del software SmartPLS 2.0 (Ringle, Wende \& Will, 2005), que emplea el método PLS-PM, un algoritmo que permite estimar los coeficientes del modelo (Esposito, Trinchera \& Amato, 2010). Los resultados sugieren dos correlaciones fuertes entre las percepciones de calidad y las satisfacción con los servicios domiciliarios, y las percepciones y la satisfacción general, esta última relacionada con la valoración de todos los servicios públicos recibidos -domiciliarios, salud, educación, seguridad, infraestructura y buen gobierno-. Estas correlaciones indican la aceptación de sólo dos hipótesis (H2 y H3), de las 4 planteadas en el modelo estructural. En la Tabla 3 se muestran las correlaciones respectivas y los valores $t$ (entre paréntesis), cuya aceptación se alcanza cuando $t>1,96$, para un nivel de confianza del 95\%. 
Tabla 1. Lista de variables observables

\begin{tabular}{|c|c|c|}
\hline Variable Latente & Variable Observable & Escala / Valoración \\
\hline \multirow{4}{*}{ Perfil del encuestado } & P1. Género & $\begin{array}{l}0 \text { Masculino } \\
1 \text { Femenino }\end{array}$ \\
\hline & P2. Edad & $\begin{array}{l}0 \text { Menores de } 20 \text { años } \\
1 \text { Entre } 20 \text { y } 40 \text { años } \\
2 \text { Entre } 40 \text { y } 60 \text { años } \\
3 \text { Más de } 60 \text { años }\end{array}$ \\
\hline & P3. Nivel de formación & $\begin{array}{l}0 \text { Primaria } \\
1 \text { Bachiller } \\
2 \text { Técnico/ Tecnólogo } \\
3 \text { Universitario } \\
4 \text { Postgrado }\end{array}$ \\
\hline & P4. Estrato socioeconómico & $\begin{array}{l}\text { Estratos socioeconómicos definidos } \\
\text { por las Leyes No } 142 \text { de } 1994 \text { y No } \\
681 \text { de 2001, de la siguiente forma: } \\
1 \text { Bajo-bajo; } \\
2 \text { Bajo } \\
3 \text { Medio-bajo } \\
4 \text { Medio } \\
5 \text { Medio-alto } \\
6 \text { Alto }\end{array}$ \\
\hline \multirow{12}{*}{ Percepciones de calidad } & P5. Disponibilidad del servicio de energía eléctrica en la ciudad & \multirow{12}{*}{$\begin{array}{l}\text { Escala Likert de } 1 \text { a } 6 \text {, siendo } 1 \text { equi- } \\
\text { valente a "muy baja valoración" y } 6 \text { a } \\
\text { "muy buena valoración". }\end{array}$} \\
\hline & P6. Facilidad para acceder al servicio de energía eléctrica & \\
\hline & P7. Disponibilidad del servicio de agua potable y alcantarillado en la ciudad & \\
\hline & P8. Facilidad para acceder al servicio de acueducto y alcantarillado & \\
\hline & P9. Disponibilidad del servicio de gas natural en la ciudad & \\
\hline & P10. Facilidad para acceder al servicio de Gas natural & \\
\hline & $\begin{array}{l}\text { P11. Disponibilidad del servicio de servicio de telefonía (fijo y/o móvil) en } \\
\text { la ciudad }\end{array}$ & \\
\hline & P12. Facilidad para acceder al servicio de telefonía & \\
\hline & P13. Disponibilidad del servicio de internet en la ciudad & \\
\hline & P14. Facilidad para acceder al servicio de internet & \\
\hline & P15. Disponibilidad del servicio de recolección de basuras en la ciudad & \\
\hline & P16. Facilidad para acceder al servicio de recolección de basuras & \\
\hline $\begin{array}{l}\text { Satisfacción con los servi- } \\
\text { cios públicos domiciliarios }\end{array}$ & P17. Grado de satisfacción general con los servicios públicos domiciliarios & $\begin{array}{l}\text { Escala Likert de } 1 \text { a } 6 \text {, siendo } 1 \text { equi- } \\
\text { valente a "totalmente insatisfecho y } 6 \\
\text { a "totalmente satisfecho". }\end{array}$ \\
\hline $\begin{array}{l}\text { Satisfacción general con los } \\
\text { servicios públicos recibidos }\end{array}$ & $\begin{array}{l}\text { P18. Satisfacción general con los servicios públicos (hospitalarios, domi- } \\
\text { ciliarios, seguridad, infraestructura, educación) de la ciudad de Cartagena }\end{array}$ & $\begin{array}{l}\text { Escala Likert de } 1 \text { a } 6 \text {, siendo } 1 \text { equi- } \\
\text { valente a "totalmente insatisfecho y } 6 \\
\text { a "totalmente satisfecho". }\end{array}$ \\
\hline
\end{tabular}

Fuente: Elaboración propia. 
Tabla 2. Resultados descriptivos de las variables observadas

\begin{tabular}{|c|c|c|c|c|}
\hline Notación & Media & Desviación & Valor Mínimo & Valor Máximo \\
\hline P1 & 0,512 & 0,501 & 0 & 1 \\
\hline P2 & 1,388 & 1,071 & 0 & 3 \\
\hline P3 & 1,936 & 0,971 & 0 & 4 \\
\hline P4 & 2,508 & 0,897 & 0 & 6 \\
\hline P5 & 3,900 & 1,305 & 1 & 6 \\
\hline P6 & 3,900 & 0,077 & 1 & 6 \\
\hline P7 & 3,964 & 0,078 & 1 & 6 \\
\hline P8 & 3,936 & 0,076 & 1 & 6 \\
\hline P9 & 4,264 & 0,083 & 1 & 6 \\
\hline P10 & 4,308 & 0,077 & 1 & 6 \\
\hline P11 & 4,148 & 0,077 & 1 & 6 \\
\hline P12 & 4,120 & 0,075 & 1 & 6 \\
\hline P13 & 4,208 & 0,080 & 1 & 6 \\
\hline P14 & 4,092 & 0,081 & 1 & 6 \\
\hline P15 & 3,888 & 0,080 & 1 & 6 \\
\hline P16 & 3,864 & 0,079 & 1 & 6 \\
\hline P17 & 3,980 & 0,074 & 1 & 6 \\
\hline P18 & 3,128 & 0,062 & 1 & 6 \\
\hline
\end{tabular}

Fuente: Elaboración propia.

Tabla 3. Correlaciones de las variables observadas

\begin{tabular}{|l|c|c|c|c|}
\hline & $\begin{array}{c}\text { Percepciones } \\
\text { de calidad }\end{array}$ & $\begin{array}{c}\text { Perfil del } \\
\text { encuestado }\end{array}$ & $\begin{array}{c}\text { Satisfacción servicio } \\
\text { domiciliario }\end{array}$ & Satisfacción general \\
\hline Percepciones de calidad & 0 & 0 & $\begin{array}{c}0,8418 \\
\mathbf{( 2 8 , 8 9 3 5 )}\end{array}$ & $\begin{array}{c}0,3537 \\
\mathbf{( 7 , 0 0 7 0 )}\end{array}$ \\
\hline Perfil del encuestado & $\begin{array}{c}0,1397 \\
(0,6230)\end{array}$ & 0 & 0 & 0 \\
\hline Satisfacción servicio domiciliario & 0 & 0 & 0 & $\begin{array}{c}0,1695 \\
(0,9402)\end{array}$ \\
\hline Satisfacción general & 0 & 0 & 0 & 0 \\
\hline
\end{tabular}

Fuente: Elaboración propia.

Las covarianzas explicadas $\left(\mathrm{R}^{\wedge} 2\right)$ de las variables latentes dependientes -Tabla 4-, indican un nivel de análisis agregado aceptable para la satisfacción sobre los servicios públicos domiciliarios, explicando su variación en un 70,86\% por las percepciones de calidad que, a su vez, no es afectada de forma significativa por el perfil de los encuestados -13,97\%, según se aprecia en la Tabla 3. Asimismo, el modelo 
demuestra una validez discriminante y homogeneidad, representada por unos valores de la Varianza Extraída Media de las variables latentes -indicador que explica en qué proporción la varianza de una variable latente es afectada por las variables observadas- superiores a las correlaciones obtenidas entre ellas mismas (Levy \& Varela, 2006), y una Fiabilidad compuesta -que mide la consistencia interna de las variables observables por cada variable latente- superior a 0,7 (Esposito, Trinchera \& Amato, 2010).

Tabla 4. Covarianzas explicadas $\left(R^{2}\right)$ y AVE de las variables latentes

\begin{tabular}{|l|c|c|c|}
\hline & $\boldsymbol{R}^{\mathbf{2}}$ & AVE & $\begin{array}{c}\text { Fiabilidad } \\
\text { Compuesta }\end{array}$ \\
\hline Percepciones de calidad & 0,019507 & 0,896400 & 0,963300 \\
\hline $\begin{array}{l}\text { Satisfacción servicio } \\
\text { domiciliario }\end{array}$ & 0,708608 & 1,000000 & 1,000000 \\
\hline Satisfacción general & 0,254841 & 1,000000 & 1,000000 \\
\hline
\end{tabular}

Fuente: Elaboración propia.

\section{Discusión y conclusiones}

El presente estudio tuvo como propósito valorar la calidad de los servicios públicos domiciliarios en la ciudad de Cartagena de Indias (Colombia), y sus efectos en el nivel de satisfacción de sus habitantes. Como valor agregado del estudio, se consideró la evaluación la influencia del perfil y percepción de la calidad del ciudadano de la ciudad de Cartagena de Indias frente a un servicio domiciliario en particular y la satisfacción respecto a la valoración general de todos los servicios públicos ofrecidos en la ciudad. Los resultados demuestran que las percepciones de calidad tienen una fuerte influencia con la satisfacción sobre los servicios públicos domiciliarios y, en menor medida, con la satisfacción general sobre los servicios públicos recibidos -aceptación de las hipótesis $\mathrm{H} 2$ y H3-. Estos resultados coinciden con diversos estudios (James, 2009; Ilhaamie, 2010; Ramseook-munhurrun et al., 2010; Rahaman \& Rahman, 2011; Kondrotaitè, 2012; Azizzadeh, Khalili \& Soltani, 2013) que indican una relación fuerte entre percepciones de calidad y satisfacción. Lo anterior permite indicar que, en parte, una mejora en las percepciones de calidad del servicio do- miciliario podría incidir positivamente en la visión general de los usuarios.

Por otra parte, con este estudio no se llegó a demostrar una relación entre el perfil del encuestado y las percepciones de calidad. Esto pudo ocurrir debido a que, en Cartagena de Indias, existe cierta homogeneidad en las problemáticas que afectan a todos los estratos socioeconómicos, sumado a la muy poca disponibilidad de empresas proveedoras de los servicios públicos domiciliarios ofertados -sólo conviven oligopolios en los servicios telefonía e internet, mientras que los demás servicios son ofertados por grandes monopolios-. Cabe anotar que en las consultas bibliográficas realizadas no se encontraron otros estudios que incluyeran el tema de perfiles como variable latente en la valoración de servicios públicos. Estas conjeturas causales indican que los gobiernos deben motivar sus esfuerzos en mejorar las condiciones de calidad, cobertura y acceso de los servicios públicos -a partir de la posterior identificación, a detalle, de los aspectos claves a mejorar por cada servicio-, para así incrementar los niveles de satisfacción en los ciudadanos. Claro está, las percepciones de calidad de un servicio público en particular afectarán en mayor medida el nivel de satisfacción sobre ese mismo servicio que la satisfacción general sobre todos los servicios recibidos.

Es de anotar que los resultados de este estudio de caso, aplicado en la ciudad de Cartagena de Indias, Colombia-, ha permitido demostrar la viabilidad del uso de la técnica de Mínimos Cuadrados Parciales -PLS- para la valoración de los servicios públicos domiciliarios, incluyendo perfiles, percepciones y satisfacción. Asimismo, su aplicación condujo a la validación de un esquema propuesto, sustentado teóricamente y empíricamente, con condiciones generales que facilitarían la evaluación de cualquier tipo de servicio público ofrecido, enfocados en dimensiones de calidad, acceso y cobertura.

Como recomendación para futuras investigaciones, se propone ampliar los campos de aplicación a otros servicios públicos, con la posibilidad de situar, en un contexto específico, la influencia que tiene el perfil del usuario en las percepciones de calidad y éstas, a su vez, valoradas en un efecto causal sobre la satisfacción general de los servicios públicos. 


\section{Referencias}

Abdi, H. (2003). Partial Least Squares (PLS) Regression. In M. S. Lewis-Beck, A. Bryman \& T. F. Liao (Eds.), Encyclopedia of Social Sciences Research Methods (pp. 1-7). Thousand Oaks: SAGE Publications Inc. http://doi. org/10.4135/9781412950589

Ackroyd, S., Hughes, J. A. \& Soothill, K. (1989). Public sector services and their management. Journal of Management Studies, 26(6), 603-619. http://doi.org/10.1111/j.1467-6486.1989. tb00747.x

Agus, A., Barker, S. \& Kandampully, J. (2007). An exploratory study of service quality in the Malaysian public service sector. International Journal of Quality \& Reliability Management, 24(2), 177-190. http://doi.org/10.1108/02656710710722284

Azizzadeh, F., Khalili, K. \& Soltani, I. (2013). Service Quality Measurement in the Public Sector (Ilam Province Post Office Case Studies). International Journal, 2(1), 114-121.

Cervantes, V. (2005). Interpretaciones del coeficiente alpha de cronbach. Avances En Medición, 3(1), 9-28.

Christensen, T. (2002). Trust in Government - the Relative importance of Service Satisfaction, Political Factors and Demography. Potsman.

Cortina, J. (1993). What is coefficient alpha? An examination of theory and applications. Journal of Applied Psychology, 78(1), 98-104. http://doi.org/10.1037/0021-9010.78.1.98

Devereux, P. (2006). Does "Satisfaction" with Local Public Services Affect Complaints (Voice) and Geographic Mobility (Exit)? Public Finance Review, 34(2), 123-147. http://doi. org/10.1177/1091142105282963

Esposito, V., Trinchera, L. \& Amato, S. (2010). PLS Path Modeling: From foundations to recent developments and open issues for model assessment and improvement. In Ja. Gentle, W. Hardle \& Y. Mori (Eds.), Handbook of Partial Least Squares: Concepts, methods and applications (pp. 47-82). London: Springer. http://doi.org/10.1007/978-3-540-32827-8

Fonseca, J. (2009). Customer satisfaction study via a latent segment model. Journal of Retailing and Consumer Services, 16(5), 352-359. http://doi.org/10.1016/j.jretconser.2009.04.001

Garthwaite, P. (1994). An Interpretation of Partial Least Squares. Journal of the American Statistical Association, 89(425), 122-127. http://doi.org/10.1080/01621459.1994.10 476452

Giannoccaro, R., Costantino, N., Ludovico, a. D. \& Pietroforte, R. (2008). Measuring Citizen Satisfaction with Aspects of Public Services from a Local Authority and Determining Their Importance: A Case Study. Public Organization Review, 8(1), 1-15. http://doi.org/10.1007/s11115-007-0044-1

Haenlein, M. \& Kaplan, A. (2004). A Beginner's Guide to Partial Least Squares Analysis. Understanding Statistics, 3(4), 283-297. http://doi.org/10.1207/s15328031us0304_4

Ilhaamie, A. (2010). Service Quality in Malaysian Public Service: Some Findings. International Journal of Trade, 1(1), 40-45. http://doi.org/10.7763/IJTEF.2010.V1.8 Ipsos MORI (2010).
Public Sector Service Satisfaction Index-A report for Consumer Focus. Londres: Consumer Focus.

James, O. (2009). Evaluating the Expectations Disconfirmation and Expectations Anchoring Approaches to Citizen Satisfaction with Local Public Services. Jnl. of Public Admininstration Research and Theory, 19(1), 107-123. http://doi. org/10.1093/jopart/mum034

Kondrotaite, G. (2012). Evaluation of the Quality of Public Services in Lithuanian Municipalities. Intelectual Economics, 6(3), 393-411.

Levy, J.-P. \& Varela, J. (2006). Modelización con estructuras de covarianzas en Ciencias Sociales. Madrid: Gesbiblo. S. L.

Mehmood, T., Martens, H., Srebø, S., Warringer, J. \& Snipen, L. (2011). A Partial Least Squares based algorithm for parsimonious variable selection. Algorithms for Molecular Biology: AMB, 6(1), 27. http://doi.org/10.1186/1748-7188-6-27

Rahaman, M. \& Rahman, A. (2011). Measuring Service Quality using SERVQUAL Model: A Study on PCBs (Private Commercial Banks) in Bangladesh. Business Management Dynamics, 1(1), 1-11.

Ramseook-munhurrun, P., Lukea-bhiwajee, S. D. \& Naidoo, P. (2010). Service quality in the public service. International Journal of Management and Marketing Research, 3(1), 37-50.

Rhee, S.-K. \& Rha, J.-Y. (2009). Public service quality and customer satisfaction: exploring the attributes of service quality in the public sector. The Service Industries Journal, 29(11), 1491-1512. http://doi.org/10.1080/02642060902793441

Ringle, C., Wende, S. \& Will, A. (2005). SmartPLS. Hamburg: SmartPLS.

Rosipal, R. \& Krämer, N. (2006). Overview and Recent Advances in Partial Least Squares. In Subspace, Latent Structure and Feature Selection Lecture Notes in Computer Science (pp. 34-51). Berlin: Springer Berlin Heidelberg. http://doi. org/10.1007/11752790_7

Swindell, D., Carolina, N. \& Kelly, J. (2005). Performance Measurement Versus City Service Satisfaction: Intra-City Variations in Quality? Social Science Quarterly, 86(3), 704-723. http:// doi.org/10.1111/j.0038-4941.2005.00325.x

Thomson, W. (2004). Customer Satisfaction With Key Public Services. CabinetOffice, 1(November), 1-7.

Van Ryzin, G. G. \& Charbonneau, É. (2010). Public Service Use and Perceived Performance: an Empirical Note on the Nature of the Relationship. Public Administration, 88(2), 551-563. http://doi.org/10.1111/j.1467-9299.2010.01820.x

Vergara-Schmalbach, J., Quesada, V. \& Blanco, I. (2011). Análisis de la calidad en el servicio y satisfacción de los usuarios en dos hoteles cinco estrellas de la ciudad de Cartagena (Colombia) mediante un modelo de ecuaciones estructurales. Ingeniare. Revista Chilena de Ingeniería, 19(3), 420-428.

Vergara-Schmalbach, J. C., Quesada, V. M. \& Blanco, I. (2012). Factores clave para la valoración de la calidad del servicio y satisfaccion del cliente: Modelos causales, desarrollo y evolución. Revista Virtual Universidad Católica Del Norte, 35(1), 380-400. 